\title{
Retardo de crecimiento intrauterino (RCIU) y sus alteraciones bioquímicas
}

\author{
Liliana Muñoz Molina M.Sc. ${ }^{1}$ y Rubinsten Hernández Barbosa M.Sc ${ }^{2}$. \\ 1. Universidad Colegio Mayor de Cundinamarca, Instituto Materno Infantil de Bogotá. 2. Gimnasio Moderno. \\ Correspondencia: cmunoz@cablenet.com.co \\ Recibido: 04 - 03 - 2005 / Aceptado: 22 - 05 - 2005 \\ Resumen
}

El estudio del retardo de crecimiento intrauterino (RCIU) continúa siendo importante tanto a nivel obstétrico como pediátrico. El interés se centra en los factores que inciden directamente en las fallas de crecimiento in útero y sobre sus consecuencias postnatales. Además del potencial genético, heredado de los padres, el desarrollo y supervivencia del feto depende directamente del suministro de los sustratos necesarios, como azúcares, ácidos grasos, proteínas, aminoácidos; de esta manera alcanza su peso potencial. Si la disponibilidad de estas fuentes disminuyen en forma persistente, la supervivencia del feto puede verse amenazada. En este artículo se hace una revisión sobre el papel que tienen dichas fuentes y sus alteraciones e implicaciones en los casos de RCIU.

\begin{abstract}
The study of the retard of intra-uterine (RCIU) growth continues being important so much at obstetric level as pediatric. The interest is centered in the factors that impact directly in the flaws of growth in uterus and on its postnatal consequences. Besides the genetic potential, inherited of the parents, the development and survival of the fetus depends directly on the supply of the necessary substrates, as sugars, fatty acid, proteins, amino acids; this way it reaches its potential weight. If the readiness of these sources diminishes in persistent form, the survival of the fetus can it turns threatened. In this article a revision is made on the paper that have this sources and its alterations and implications in the cases of RCIU.
\end{abstract}

\section{Introducción}

El RCIU se define como aquella circunstancia clínica en la cual el feto no alcanza su pleno potencial de crecimiento; como resultado final ocurre una disminución en el peso corporal, el cual queda por debajo del percentil 10 para la edad gestacional según tablas de crecimiento (1). Aunque la mayoría de los niños que nacen con RCIU alcanzan una talla adecuada, especialmente durante el primer año de vida (2), aproximadamente entre un $15-20 \%$ presentan talla baja (3).

Piel laxa, delgada, tejido graso subcutáneo reducido, abdomen excavado y grasa muscular de brazos, glúteos y muslos disminuida son características físicas de los neonatos con RCIU. Esta patología se considera una condición multifactorial donde están incluidos aspectos fisiopatológicos fetales, placentarios y maternos, entre los que se destacan reducción de nutrientes y disponibilidad de oxígeno, ingestión de drogas, disminución de la masa y flujo placentario, infección congénita y anomalías cromosómicas (4). El RCIU está asociado con un incremento en la morbilidad y mortalidad fetal antes y después del nacimiento $(5,6)$. Basándose en el índice ponderal el RCIU puede presentarse bajo dos formas, RCIU simétrico y RCIU asimétrico (7). 


\section{RCIU y Glucosa}

La glucosa es el principal substrato para el metabolismo energético fetal y su demanda se incrementa a medida que avanza el crecimiento. $\mathrm{Su}$ transporte, dado a través de la placenta, depende de factores como el flujo sanguíneo, densidad del transportador y cambios en el potencial de membrana (8). Mediciones de glucosa en sangre obtenida por cordocentesis en fetos con RCIU han reportado concentraciones bajas, indicando que el feto humano con RCIU es hipoglicémico. Esto podría ser el resultado de alteraciones en la placenta o del metabolismo de la glucosa fetal (9). Niveles bajos de glucosa también han sido encontrados en niños con RCIU cuyas madres presentan niveles normales, sugiriendo disminución de la transferencia de glucosa a través de la placenta, presumiblemente debido a los transportadores de glu$\operatorname{cosa}(10,11)$.

\section{RCIU y Aminoácidos}

El consumo de etanol, nicotina, morfina y cocaína afecta notablemente el transporte de aminoácidos de la placenta al feto. Una disminución en la transferencia de aminoácidos a través de la placenta, o una dieta baja en proteínas, puede ser causa de RCIU (12). En sangre de cordón de fetos con RCIU se ha encontrado concentraciones bajas de aminoácidos esenciales como lisina y leucina, que son transportados a través de la placenta por el sistema de transporte independiente de sodio (13).

Los aminoácidos presentes en la circulación materna son transportados por la placenta hacia la circulación fetal. Sin embargo, no todos los aminoácidos que entran a la circulación fetal desde la placenta son originados en la circulación materna, algunos son sintetizados dentro de ésta a partir de otros precursores. Eventualmente un aminoácido de la circulación materna puede ser utilizado en su totalidad dentro de la placenta (14). Los aminoácidos no esenciales como la glicina y la alanina son transportados por el sistema dependiente de sodio. La glicina se encuentra en concentraciones elevadas en la placenta y puede ser importante en la absorción de otros aminoácidos (15).

\section{RCIU y Lípidos}

Los ácidos grasos son componentes fundamentales de la membrana celular y son importantes para el desarrollo y crecimiento fetal; son fuente de energía y actúan como precursores de moléculas de señalización celular (16). Estudios recientes sobre el rol de la oxidación de los ácidos grasos en la unidad fetoplacentaria han demostrado que la placenta expresa enzimas importantes para el proceso de $\beta$-oxidación de ácidos grasos de cadena larga, comparables a las presentes en el músculo esquelético (17). Cuando no se produce la ß-oxidación, hay una transferencia de metabolitos intermediarios a la circulación materna, quienes han sido postulados ser causantes de preclampsia, lo que a su vez puede causar RCIU (18).

Se ha asociado una alteración en el metabolismo de lípidos con disminución de los depósitos de grasa. En plasma fetal se ha encontrado que los niveles de ácidos poliinsaturados de cadena larga (araquidónico y decosahexanoico) y sus metabolitos, también como sus precursores, los ácidos linoleico y el á linoleico (19) están significativamente disminuidos comprados con los maternos (20). Estos ácidos son necesarios en el desarrollo fetal especialmente por su alta demanda en la retina y el cerebro, cuyo máximo crecimiento se da entre el último trimestre de embarazo y los primeros meses de vida postnatal (21). Esta disminución ha sido asociada a complicaciones vasculares y neuronales, como también a hemorragias intraventriculares y retinopatías en la vida postnatal (22).

Se han informado concentraciones altas de triglicéridos y ácidos grasos libres disminuidos en fetos con RCIU. Estas alteraciones son dadas por hipoglicemia crónica con lipólisis compensatoria e inhabilidad para hidrolizar los triglicéridos circulantes permitiendo la disminución del uso de los mismos para el depósito de 
grasa (23). Análisis en sangre de cordón de niños con RCIU, determinaron un aumento de la relación: lipoproteínas de alta densidad (HDL)/apolipoproteina A y apolipoproteina $\mathrm{B}$ /apolipoproteina $\mathrm{A}$, debido a la disminución de los niveles de apolipoprotiena A producida por el hígado fetal, sugiriendo una alteración en la síntesis hepática o en la secreción (24).

Por otro lado, la leptina, hormona peptídica expresada y secretada por los adipositos, es conocida por regular el crecimiento del cuerpo y la masa de tejido adiposo a través de un mecanismo de retroalimentación (25). Su expresión en la placenta humana y en el amnios sugiere que puede estar involucrada en el crecimiento y desarrollo fetal (26). Durante el embarazo los niveles de leptina en sangre materna aumentan a medida que este transcurre, para luego descender después del nacimiento (27). Las concentraciones de leptina en liquido amniótico y sangre de cordón han sido correlacionadas con el peso al nacer, siendo sus niveles más bajos en los casos de RCIU $(28,29)$.

\section{RCIU y Minerales}

El rol de los minerales sobre el crecimiento fetal ha sido menos estudiado. Los minerales pueden directamente afectar el crecimiento lineal y ponderal, como también pueden tener efectos indirectos ya que actúan como cofactores para las enzimas, las cuales se unen a dominios del ADN o actúan como componentes esenciales de la matriz ósea (30). Las reservas de zinc y de hierro en el feto son importantes para contribuir en un adecuado crecimiento. Concentraciones bajas de Zinc y de Hierro durante el embarazo se han asociado a un crecimiento fetal disminuido y bajo peso al nacer (31).

El Zinc, el elemento más abundante intracelularmente, es necesario para la formación de RNA polimerasa I, II, III y ayuda a la configuración de las proteínas y de las metaloenzimas (32). El hierro se encuentra formando parte de las proteínas, especialmente de la hemoglobina que contiene el $60 \%$ de hierro en el cuerpo, y de las enzimas mitocondriales, las cuales están involucradas en la síntesis de ATP. La deficiencia de hierro y la anemia durante el embarazo incrementa el riesgo de tener niños con bajo peso. Se considera que la transferencia del hierro al feto puede estar regulado por la reserva de la madre, quien debe transferir aproximadamente el 15\% durante el periodo de gestación (33).

La deficiencia de hierro afecta potencialmente procesos de desarrollo cerebral como la mielinización (34), el metabolismo monoamínico, el metabolismo energético y el crecimiento dendrítico del hipocampo (35). La suplementación de estos minerales durante el embarazo y los primeros meses de vida postnatal en niños con bajo peso al nacer y RCIU, ha resultado en un incremento significativo de peso y en la disminución de su vulnerabilidad, comparado con niños que no recibieron estos suplementos (36).

Otro elemento importante que ha sido objeto de estudio en los casos de RCIU es el magnesio ( $\mathrm{Mg}$ ), que forma parte estructural de los huesos, tejidos blandos y dientes, constituyéndose en el quinto mineral en abundancia en el organismo. Un estudio prospectivo, comparando las concentraciones de $\mathrm{Mg}$ en sangre de cordón, y de neonatos a los tres y siete días de vida, reportó concentraciones más bajas en los casos de RCIU comparados con los adecuados para la edad gestacional. Lo anterior sugiere que este ión puede tener un efecto sobre la modulación de la homeostasis durante el periodo perinatal (37).

\section{RCIU y Los IGFs}

El crecimiento fetal in útero está influenciado por factores genéticos, nutricionales y hormonales. De la compleja red de factores de crecimiento y hormonas, que participan y controlan este proceso, los IGFs tienen un papel fundamental, no solo por estimular el anabolismo y la proliferación celular, sino también por su capacidad de distribución de nutrientes entre la placenta y el feto, a favor del feto (38).

Aunque se conoce el rol del sistema IGF (IGF-I, IGF-II y sus receptores), pocos casos de RCIU han 
sido atribuidos a anormalidades genéticas de este sistema, cuyos defectos están a nivel cromosomal asociados a otras anormalidades, entre las cuales figuran retraso en el desarrollo, hipoplasia de órganos y braquidactilia entre otros (39). Se ha encontrado que el receptor tipo I (IGF-IR) tiene una concentración menor en los casos de RCIU y su afinidad por el IGFI está disminuido, especialmente en los eritrocitos, aunque éstos tengan un gran número de receptores comparado con los niños de peso adecuado para la edad gestacional (40). En los embarazos complicados por RCIU se ha podido establecer que las placentas presentan una disminución en la concentración del IGF-IR, lo cual puede contribuir a la alteración de las vías de señalización de este importante factor de crecimiento (41).

Especialmente el factor de crecimiento insulínico (insulin-like growth factor-I, IGF-I), ha mostrado ser de mayor relevancia en la caracterización del fenómeno del crecimiento intrauterino, es producido por la mayoría de los tejidos fetales, actúa de manera paracrina induciendo el depósito de nuevo tejido. En sangre fetal y liquido amniótico ha mostrado relación con el peso fetal $(42,43)$. La biodisponibilidad del IGFI está regulada por seis proteínas de unión (IGFBPs). El $80 \%$ de lGF-I en circulación se encuentra formando un complejo ternario de $150 \mathrm{kDs}$ con la IGFBP3 y una subunidad ácido lábil (44).

En recién nacidos e infantes con RCIU, se han descrito dos proteasas, que convierten la molécula de IGFBP-3 en dos fragmentos diferentes, permitiendo eventualmente el incremento del IGF-I (45). En sangre materna, de mujeres con fetos con diagnóstico de RCIU, se ha observado que los niveles de la IGFBP3 aumentan en el primer y segundo trimestre, para disminuir en el tercero, probablemente por el efecto de la insulina sobre el IGFBP-1 (46).

La IGFBP-1, mediante la unión al IGF-I regula la disponibilidad de IGF-I libre en circulación (47) y sus niveles en sangre fetal, tomada por cordoncentesis, especialmente en el tercer trimestre de gestación, han sido más altos en los casos de RCIU (48). También la concentración de cortisol se ha correlacionado positivamente con la IGFBP-1 y negativamente con el IGF-I en recién nacidos con peso apropiado para la edad gestacional. Sin embargo, en los casos de RCIU se ha encontrado una relación inversa entre los valores de cortisol en plasma y la longitud ganada en el primer trimestre de vida (49). Además, se ha informado una relación inversa entre los niveles de cortisol y el peso y longitud al nacer, y una relación alta entre cortisol/ cortisona, sugiriendo una deficiencia parcial de 11ßhidroxiesteroide deshidrogenasa tipo 2. Lo anotado anteriormente ha permitido sugerir que el cortisol podría modular negativamente modular el crecimiento durante el período perinatal en los niños con RCIU (50).

\section{RCIU y Liquido Amniótico}

La composición y el volumen del liquido amniótico está regulado por muchos procesos, los cuales incluyen: producción a través de la orina fetal y fluido pulmonar, remoción por deglución fetal y transferencia intramembranosa hacia el feto, y en menor proporción transferencia a través de la membrana coriónica hacia la madre (51). Otro de los hallazgos asociados al RCIU es el oligohidramnios, que se presenta en el $40 \%$ de los casos (52). La causa más probable es la reducción del flujo urinario fetal, como consecuencia de la redistribución del flujo sanguíneo, con desviación preferente hacia el cerebro y disminución de la perfusión renal (53). También puede ser causada por una insuficiencia placentaria, como producto de una hipoxia fetal, lo cual hace que se incremente la osmolaridad, las concentraciones de sodio y la reabsorción de agua por los tubulos renales (54).

\section{RCIU y Vida Postnatal}

Los niños nacidos con RCIU en la etapa adulta son más susceptibles de presentar enfermedades cardiovasculares, hipertensión, dislipidemias, obesidad (55), menarquia precoz e infertilidad (56), diabetes mellitus no insulinodependientes (57) y son más vulnerables a las infecciones. Tienen características fisiológicas que se cree 
alteran el desarrollo y metabolismo en la función de órganos. Algunos estudios reportan afección al sistema nervioso central (58). Las consecuencias del RCIU han sido estudiadas en tres campos:

- La morbilidad y mortalidad perinatal. Los infantes nacidos con RCIU presentan mayores niveles de morbilidad y mortalidad perinatal, comparados con los niños de apropiado peso para la edad gestacional (59).

Crecimiento y desarrollo del niño. Aunque en el primer año de vida hay un crecimiento marcado, la gran mayoría permanecen más pequeños hasta el final de la adolescencia.

- Salud y bienestar en las etapas de adolescencia y adulta. Se han encontrado diferencias en el coeficiente intelectual, dificultades en el lenguaje y problemas de comportamiento entre los niños y adolescentes que nacieron con RCIU (60).

Las consecuencias del RCIU en la adolescencia y en la etapa adulta, sobre el crecimiento y desarrollo cognoscitivo han sido menos estudiadas y entendidas, debido a su gran complejidad y a lo dispendioso del seguimiento de quienes forman parte del estudio. Sin embargo, algunos estudios que hay al respecto, han cobijado los siguientes aspectos:

El cerebro: Existe un período de sensibilidad en el proceso de maduración del cerebro antes y después del nacimiento (61), durante el cual el crecimiento, al igual que el comportamiento y desarrollo cognoscitivo pueden ser alterados por un déficit importante de nutrientes (62). Empleando resonancia magnética tridimensional se determinó que fetos con diagnóstico de RCIU, tenían el volumen cerebral disminuido, sugiriendo daño a nivel estructural, especialmente sobre la zona cortical (63).

Comportamiento del niño: Los niños con RCIU generalmente tienen desarrollo sicomotor más lento, son más callados y difíciles de motivar que niños normales, presentan un estado de sueño inmaduro (64), su llanto es inusual, con frecuencias altas y poco armonioso, y su fijación visual está disminuida. En el primer año de vida su sistema neurológico es menos maduro comparado con los de peso apropiado para la edad gestacional (65).

A manera de síntesis, es importante anotar que el potencial adverso causado por la falta de metabolitos necesarios, al igual que la vulnerabilidad de un órgano y/o sistema particular en el feto, depende de la clase de nutrientes, la concentración y el tiempo de carencia de los mismos. Según el período gestacional, los efectos que desencadenan la carencia y/o déficit de los sustratos analizados en este articulo, pueden alterar procesos morfológicos o la expresión de genes para proteínas o factores endocrinos importantes en el crecimiento y desarrollo fetal y postnatal.

\section{Referencias}

1. Lubchenco H, Hansman C, Dressler M, Boyd R. Intrauterine growth as estimated from liveborn birth weight data at 24 to 42 wk of gestation. Pediatrics 1963; 32:793-800.

2. Albersson-Wikland K, Karlberg J. Natural growth in children born small for gestation age with and without catch-up growth. Acta Pediatr 1994; 399(Suppl1):64-70.

3. Karlberg J, Albertsson-Wikland K. Growth in full-term smallfor-gestation-age infants: from birth to final height. Pediatr Res 1995; 38:733-739.

4. Gembruch, U. and Gortner, L.. Perinatal aspects of preterm intrauterine growth restriction. Ultrasound in Obstet Gynecol. Ed. 1999; 233-239.

5. Piper, J, Xenakis, E, McFarland, M, Elliot, B, Berkus M and Langer $O$. Do growth-retarded premature infants have different rates of perinatal morbidity and mortality than appropriately grown premature infants? Obstet Gynecol. 1996; 87:169-174.

6. Garite T, ClarK R, Thorp J. Intrauterine Growth restriction increases morbility and mortality among premature neonates. Am J Obstet Gynecol. 2004; 191:481-487.

7. Brooks A, Johnson M, Pawson M and Abdala H. Birthweigth: nature or nurture? Early Human. Devel 1995;42:29-35.

8. Gluckman, P. Endocrine and nutritional regulation of prenatal growth.. Acta Pediat Suppl 1997; 423:153-157.

9. Karsdorp V and Van J. Amino acids, glucose and lactate concentration in umbilical cord blood in relation to umbilical artery flow patterns. Eur. J Obstet Ginecol 1994; 57:117122.

10. Nicolini U, Nicolaides P, Fisk N, Vaughn J, Fusi L, Gleeson R et al. Limited role of fetal blood sample in prediction of outcome in intrauterine growth retardation Lancet 1990; 336:768-772

11. Thamotharan M, Shin B, Suddirikku T, Thamotharan SH, Garg $M$ and Devaskar S. GLUT4 expression and subcellular localization in the intrauterine growth-restricted adult rat female offspring. Am J Physiol Endocrinol Metab 2005; 288: E935-E947.

12. Cetin I, Corbetta C, Sereni L, Marconi A, Bozzetti P, Pardi G and Battaglia F. Umbilical amino acid concentration in normal and growth retarded fetuses sample in utero by cordocentesis. American. J Obstet Gynecol. 1990; 162:253261 . 
13. Marconi A, Cetin I, Buscaglia M. and Pardi G. Mid gestation cord sampling: what have we learned?. Placenta 1992; 13:115122 .

14. Sibley C, Glazier J and D'Sovza S. Placental transporter activity an expression and relation to fetal growth. Exp Physiol. 1997; 82:3989-402.

15. Cetin I, Marconi A, Baggiani A, Buscaglia M, Pardi G, Fennessey, $\mathrm{P}$ and Battaglia F. In Vivo Placental transport of Glycine and leucine in human pregnancies. Pediatr Res 1995; 37:571-575.

16. Oey N, Den M, Vekemans M, Auge J, Wijburg F, Steiner C et al. Long-chain fatty acid oxidation during early human development. Pediatr Res 2005; 57:755-759.

17 Oey N, den Boer M, Ruiter J, Wanders R. Duran M, Waterhan H, Boer K, Vander J, Wijburg F. High activity of fatty acid oxidation enzymes in human placenta: implications for fetal-maternal disease. J Inherit Metab Dis 2003; 26:385-392.

18 Shekhawat P, Matern D and Staruss A. Fetal fatty acid oxidation disorders, their effect on maternal health and neonatal outcome: Impact of expanded newborn screening on their diagnosis and management Peadiatr Res 2005; 57: 78R-86R.

19. Vilbergssson G, Samsioe G, Wennergren M, Karlsson K. Essential fatty acids in pregnancies complicated by intrauterine 20. Giovanni M, Riva E, Agostoni C. Fatty acids in pediatric nutrition. Pediatr Clin North Am. 1995; 42:861-877.

21. Cetin I, Giovannini N, AlvinoG, Agostoni C, Riva E, Giovanini $M$ and Pardi $G$. Intrauterine growth restriction is associated with changes in polyiunsaturated fatty acid-maternal relationships. Pediatr Res 2002; 52:750-755.

22. Innis SM. Perinatal biochemistry and physiology of long chain polyunsaturated fatty acids J Pediatr 2003; 143:S1-S8.

23. Sivan E, Whittaker P, Sinha D, Homko C, Lin M, Reece E and Boden G. Leptin in human pregnancy: relationship with gestacional hormones. Am J Obstet Gynecol 1998; 179:11281132 .

24. Jones, J., Gercel-Taylor, C. and Taylor, D. Altered cord serum lipid levels associated with small for gestational age infants. Obstet Gynecol 1999; 93:527-531.

25. Zhang B, Graziano M and Doebber T. Down-regulation of the expression of the obese gene by an antidiabetic thiazolidinedione in Zucker diabetic fatty rats and $\mathrm{db} / \mathrm{db}$ mice. J Biol Chem. 1996; 271:9455-9459.

26. Hoggard N, Hunter L, Duncan J, Williams L, Trayhurn P and Mercer J. Leptin and leptin receptor mRNA and protein expression in the murine fetus and placenta. Proc Natl Acad Sci USA 1997; 94:11073-11078.

27. Masuzaki H, Ogawa Y, Sagawa N, Hosada K, et all. Nonadipose tissue production of leptin: leptin as a novel placenta-derived hormone in human. Obstet Gynecol Survey 1998; 53:156158 .

28. Schubring C, Kiess W, Englaro P, Rascher W, Dotsch J et all. Levels opf leptin in maternal serum, amniotic fluid and arterial and venous cord blood: relation to neonatal and placental weight. J Clin Endocrinol Metab 1998; 82:1480-1483.

29. Muñoz L, Sabogal J, Arteaga C., Hernández R, Mockus I, Tovar J. Dterminación perinatal de leptina en madres y neonatos con diagnóstico de retardo de crecimiento intrauterino (RCIU). Nova 2003; 1:49-56.

30. O'Brien, K.. Regulation of mineral metabolism from to infant: metabolic studies. Acta Paediatr Suppl 1999; 433:88-91.

31. Rivera J, Ruel M, Santizo M, Lonnerdal B, Brown K. Zinc supplementation improves the growth of stunted rural Guetamelan infants. J Nutr 1998; 128:556-562.

32. Wiliams, R. Zinc: what is its role in biology?. Endeavour 1984; 8:65-70.

33. Dallman P. Changing iron needs from birth through adolescence. In: Fomon SJ, Zlotkin S, editors. Nutrional anemias. New York: Raven Press 1992; 29-36.
34 Connor J, Menzies S. Altered cellular distribution of iron in the central nervous system of myelin deficiente rats Neuroscience 1990; 34: 265-271.

35. Jorgenson L, Wobken J, and Gerogielf M. Perinatal iron defienciency alters apical dendritic growth in hippocampal CA-1 pyramidal neurons. Develop Neuroscience 2003; 25:412-420.

36. Krebs N, Bartlett A, Westcott $J$, et al. Exchangeable zinc pool size is smaller at birth in small for gestational age infants. Pediatr Res 2003; 53: 394.

37. Barbosa N, Okay T and Leone C. Magnesium and Intrauterine Growth Restriction A Journal of the American College of Nutrition 2005; 24:10-15.

38. Siebler T, Lopaczynski W, Terry C, Casella S, Munson P, et al. Insulin-like growth factor I receptor expression and function in fibroblasts from to patients with deletion of de distal long arm of chromosome 15. J Clin Endocrinol Metab 1995; 80:3447-3457.

39. Ducos B, Cabrol S, Houang M, Perin L, Holzenberger M, Le Bouc Y. IGF type 1 receptor ligand binding characteristics are altered in a subgroup of children with intrauterine growth retardation. J Clin Endocrinol Metab 2001; 86:5516-5524.

40. Laviola L, Perrini S, Belsanti G, Natalicchio A, Montrone C, Leonardini A, Vimercati A, Scioscia M, Selvaggi L, Giorgino $\mathrm{R}$, Greco $\mathrm{P}$ and Giorgino F. Intrauterine Growth Restriction in Humans Is Associated with Abnormalities in Placental Insulin-Like Growth Factor Signaling . Endocrinol 2005; 146:1498-1505.

41. Sabogal J, Hernández R, Arteaga C, Muñoz L. Relación entre los niveles de IGF-I y la presencia o ausencia de retardo de crecimiento intrauterino (RCIU). Saludarte 2003; 9:7-24.

42. Verkauskiene R, Jaquet D., Deghmoun S, Chevenne D, Czernichow P, and Lévy-Marchal C. Smallness for Gestational Age Is Associated with Persistent Change in Insulin-Like Growth Factor I (IGF-I) and the Ratio of IGF-I/IGF-Binding Protein-3 in Adulthood J Clin Endocrinol Metab 2005; 90:5672-5676.

43 Gohlke B, Huber A, Hecher K, Fimmers R, Bartmann P and Roth C. Fetal Insulin-Like Growth Factor (IGF)-I, IGF-II, and Ghrelin in Association with Birth Weight and Postnatal Growth in Monozygotic Twins with Discordant Growth J Clin Endocrinol Metab 2005; 90:2270-2274.

44. Han, V., Bassett, N., Walton, J. and Challis J. The expression of insulin-like growth factor (IGF) and IGF-binding protein (IGFBP) genes in the human placenta and membranes: evidence for IGF-IGFBP interactions at the feto-maternal interface J Clin Endocrinol Metab 1996; 81:2680-2693.

45. Cianfarini S, Germani D, Rossi P, Rossi L, Germani A, Ossicini $\mathrm{C}$, et al intrauterine growth retardation: evidence for the activation of the insuline growth factor (IGF) related growthpromoting machinery and the presence of a cationindependent IGF binding protein-three proteolytic activity by two months of live. Pediatr Res 1998; 44:374-380.

46. Bhatia S, Faessen G, Carland G, Balise L, Gargosky S, Druzin $\mathrm{M}$, et al. A longitudinal analysis of maternal serum insulinelike growth factor I (IGF-I) and total and nonphosphorylated IGF-binding protein-1 in human pregnancies complicared by intrauterine growth restriction. J Clin Endocrinol Metab 2002; 87:1864-1870.

47. Gibson J, Aplin J, White A, Westwood M. Regulation of IGF bioavailability in pregnancy. Mol Hum Reprod 2001; 7: 79-87.

48. Langford K, Nicolaides $\mathrm{K}$ and Miell J. Maternal and fetal insulin-like growth factor and their binding protein in the second and third trimesters of human pregnancy. Hum Reprod 1998; 13:1389-1393.

49. Cianfarani S, Germani D, Rossi L, Argiro G, Boemi S, Lemon M, et al. IGF-I and IGF-binding protein-I are retalted to cortisol in human cord blood. Eur J Endocrinol 1998; 138:524-529. 
50. Cianfarani S, Geremia C, Scott C, Germani D. Growth, IGF System, and cortisol in children with intrauterine growth retardation: is catch-up growth affected by reprogramming of the hypothalamic-pititary-adrenal axis? Pediatr Res 2002; 51:94-99.

51. Brace, R. and Moore, T. Transplacental, amniotic, urinary, and fetal fluid dynamics during very large volume fetal intravenous infusions. Am J Obste Gynecol 199; 164:907916.

52. Philpson, E., Sokol, R. and William, T. Oligohydramnios: clinical associations and predictive value for intrauterine growth retardation. Am J Obstet Gynecol. 1983; 146:271.

53. Polin, R. and Fox, W. Fetal and Neonatal physiology. WB Saunders 1992.

54. Salha, S., Raymond, I., Pamela, J. and Stanley, J. Amniotic fluid composition in the fetal lamb with intrauterine growth restriction. Am. J Obstet 1999; 180:703-710.

55. Barker D, Gluckman P, Godfrey K, Harding J, Owens J, Robinson J. Fetal Nutrition and cardiovascular disease in adult life. Lancet 1993; 341:938-941

56. Ibáñez L, Potau N, de Zegher F. Recognition of a new association: reduced fetal growth, precocious pubarche, hyperinsulinism and ovarian dysfunction. Ann Endocrinol (Paris) 2000; 61:141-142

57. Barker D, Hales C, Fall C, Osmond C, Phipps K, Clark P. Type 2 (non-insulin dependent) diabetes mellitus, hypertension and hyperlipidemia (syndrome $\mathrm{X}$ ): relation to reduced fetal growth. Diabetologia 1993; 36:62-67.

58. Blair, E. and Stanley, F. Intrauterine growth and spastic cerebral palsy. I. Association with birth weight for gestational age. Am J Obstet Gynecol. 1990; 162:229-237.

59. Garite T, Clark R, Thorp J. Intrauterine growth restriction increases morbidity and mortality among premature neonates. Am J Obstet Gynecol 2004; 191:481-487.

61. Foulder-Hughes L and Cooke RWI. Motor, cognitive and behavioural disorders in children born very preterm. Dev Med Child Neurol 2003; 45:97-103.1B

62. Lucas A, Morley R, Cole T, Lister G and Leeson-Payne C. Breast milk and subsequent intelligence quotient in children born pre term. Lancet 1992; 339:261-264.

63. Borradori C, Zimine S, Warfield S, Freschi M, Sancho A, et al. Early alteration of structural and functional brain development in premature infants born with intrauterine growth retardation. Pediatr Res 2004; 56:132-138.

64. Pryor, J. The identification and long term effects of fetal growth restriction. British J Obstet Gynecol 1996; 103:11161122 .

65. Ounsted, M, Moar V and Scott A. Neurological development of small-for gestational-age babies during de first year of life. Early Hum. Dev 1988; 16:166-172. 